\title{
Anästhesie bei Erwachsenen mit Adipositas
}

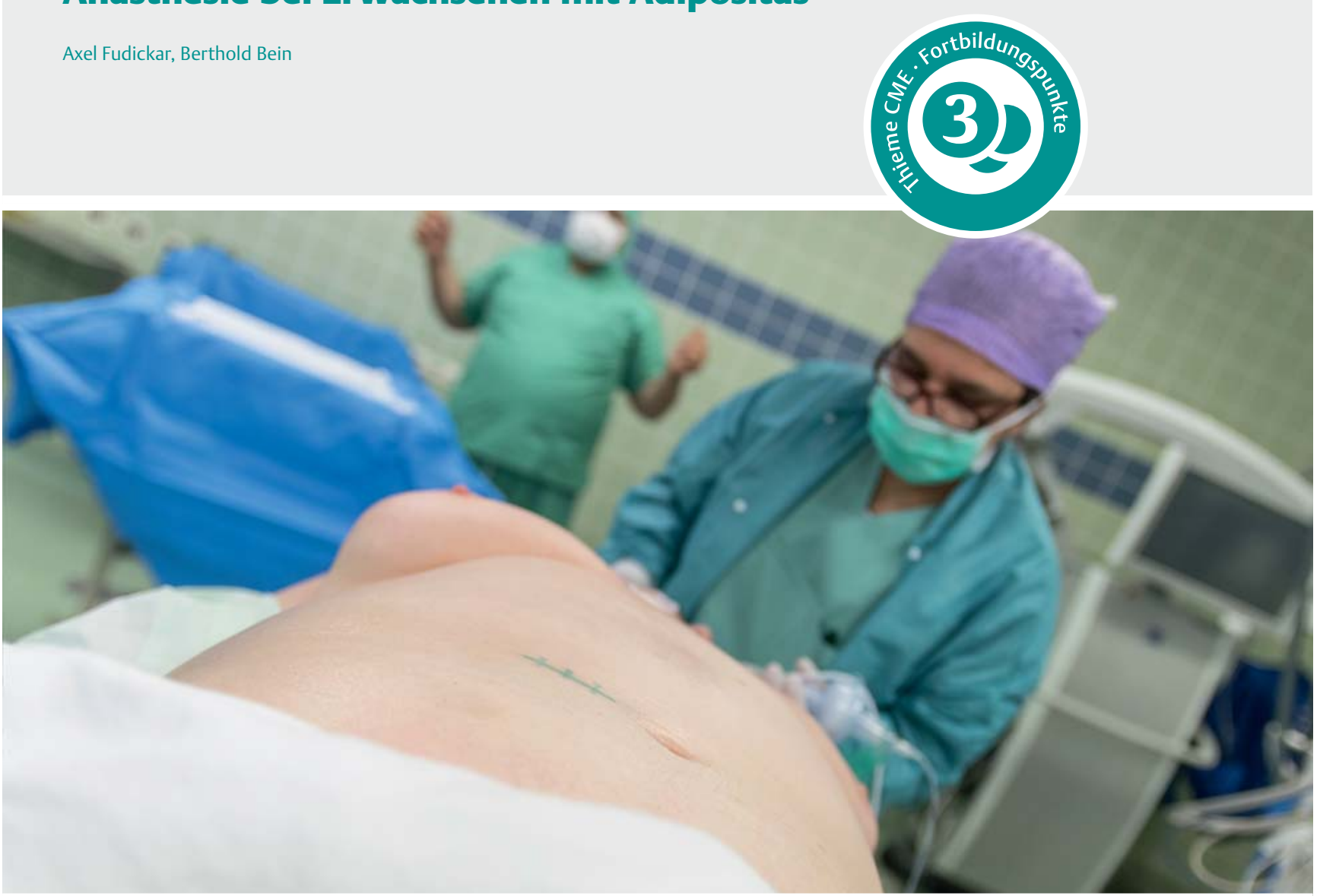

Quelle: KH Krauskopf

Die Prävalenz der Adipositas hat in den letzten 10 Jahren weltweit erheblich zugenommen - deshalb wird die perioperative Versorgung auch extrem adipöser Patienten immer häufiger notwendig werden. Dies schließt medizinische, technische und organisatorische Aspekte ein. Dieser Beitrag fasst neue Erkenntnisse und Empfehlungen zur Anästhesie bei Adipositas zusammen.

\section{Einleitung}

\section{Definition und Einteilung der Adipositas}

Nach WHO-Definition ist Adipositas als ein Übermaß an Körperfett definiert, das zu unerwünschten Folgen für Gesundheit und Wohlbefinden führt [1].

Beim androiden Typus, dem bei Männern häufigeren Typ der Adipositas, überwiegt eine zentrale (stammbezogene) Fettverteilung (auch als Apfel-Typ oder Falstaff-Typ nach Shakespeares John Falstaff - bezeichnet). Die Aus- prägung des androiden Typs kann quantifiziert werden durch

- das Verhältnis von Taillenumfang zur Körpergröße (waist to height ratio, WTHR, pathologisch ab 0,55 ) oder

- den absoluten Taillenumfang (waist circumference, WC, pathologisch ab $102 \mathrm{~cm}$ bei Männern und $88 \mathrm{~cm}$ bei Frauen).

Der gynoide, bei Frauen häufigere Typ ist durch eine periphere (gliedmaßenbezogene) Fettverteilung gekennzeichnet (auch als Birnen-Typ oder Rubens-Typ bezeichnet). 


\section{Quantifizierung}

Der Body-Mass-Index (BMI) ist das bekannteste Maß zur Quantifizierung von Übergewicht und Adipositas:

Body-Mass-Index $($ BMI $)=\frac{\text { Gewicht }[\mathrm{kg}]}{(\text { Größe }[\mathrm{m}])^{2}}$

Übergewicht liegt nach Definition der WHO ab einem BMI von $25 \mathrm{~kg} / \mathrm{m}^{2}$ vor. Adipositas Grad I ist als ein Übergewicht mit einem BMI von mehr als $30 \mathrm{~kg} / \mathrm{m}^{2}$ definiert. Adipositas Grad II und III werden durch einen BMI von über $35 \mathrm{~kg} / \mathrm{m}^{2}$ und über $40 \mathrm{~kg} / \mathrm{m}^{2}$ abgegrenzt.

Cave

Der BMI kann bei muskulösen Menschen falsch positive Werte für Adipositas ergeben.

\section{Prävalenz}

Die Prävalenz der Adipositas beträgt in Deutschland 23\% für Frauen und 24\% für Männer. 67\% der Männer und $53 \%$ der Frauen sind übergewichtig. Im weltweiten Vergleich scheint sich die Zunahme des durchschnittlichen Körpergewichts in westlichen Ländern und asiatischen Ländern mit hohem Einkommen eher zu verlangsamen. Dahingegen steigt sie in südostasiatischen Ländern und anderen Schwellenländern eher an.

\section{Ätiologie}

Die Ursache für Übergewicht und Adipositas ist ein Missverhältnis zwischen Energiezufuhr und Energieverbrauch. Meistens führt Bewegungsmangel in Kombination mit energiereicher Ernährung dazu, dass nicht verbrauchte Energie als Fett gespeichert wird. Deutlich seltenere Ursachen des Übergewichts sind Stoffwechselerkrankungen.

\section{Merke}

Das Risiko einer Entwicklung von Adipositas ist schon im Kindesalter von einer Reihe adipositasrelevanter genetischer und psychosozialer Einflussfaktoren z. B. ökonomischer Status und Ernährungsbewusstsein - abhängig [2].

\section{Folge- und Begleiterkrankungen}

- Tab. 1 zeigt die anästhesierelevanten Folge- und Begleiterkrankungen der Adipositas, auf die nachfolgend detailliert eingegangen wird.

\section{Obstruktives Schlafapnoe-Syndrom}

Das Schlafapnoe-Syndrom (obstruktives SchlafapnoeSyndrom, OSAS) wird diagnostiziert, wenn mehr als 5 Apnoe- oder Hypopnoe-Phasen pro Stunde Schlaf in Kombination mit Tagesmüdigkeit auftreten. Diese Apnoe-Phasen führen zu passagerem Sauerstoffmangel und zu Tagesmüdigkeit. Ein OSAS hat eine erhöhte Inzidenz von respiratorischen und kardiovaskulären intra- und postope-
- Tab. 1 Folge- und Begleiterkrankungen der Adipositas.

\begin{tabular}{|l|l|}
\hline Organsystem & anästhesierelevante Begleiterkrankungen \\
\hline Atemwege & - schwieriger Atemweg \\
\hline Beatmung/Atmung & - Schlafapnoe-Syndrom \\
\hline - Obestriktive und obstruktive Atemstörung \\
\hline Herz & - koronare Herzkrankheit \\
\hline & - Herzrhythmusstörungen \\
\hline Kreislauf & - arterielle Hypertonie \\
\hline Gehirn und Nerven- & - psychiatrische Erkrankungen \\
\hline system & - zerebrale Ischämie \\
\hline Bewegungsapparat & - Arthrose \\
\hline Gerinnung & - Arthritis \\
\hline Immunsystem & - Thrombtionelle Beschwerden \\
\hline Gastrointestinaltrakt & - Vitamin-K-Mangel \\
\hline Leber und Gallewege & - chronische Entzündungsreaktion \\
\hline Stoffwechsel & - gastroösophagealer Reflux \\
\hline & - relative Mangelernährung \\
\hline & - nicht alkoholbedingte Fettleber \\
\hline & - Cholezystolithiasis \\
\hline & - Hyperlipidämie \\
\hline & - Diabetes mellitus \\
\hline
\end{tabular}

rativen Komplikationen zur Folge (s. „Fallbeispiel - Schlafapnoe-Syndrom“).

\section{FALLBEISPIEL}

\section{Schlafapnoe-Syndrom}

Eine 37-jährige adipöse Patientin (Gewicht 193 kg, Größe $173 \mathrm{~cm}$, Nikotinkonsum ca. 20 Zigaretten proTag, regelmäßiger Alkoholkonsum) wird im Nachtdienst mit Gallenkolik zur laparoskopischen Gallenblasenresektion angemeldet. Wegen Schlafstörungen nimmt die Patientin regelmäßig Zolpidem und Lorazepam $\left(\right.$ Tavor $\left.^{\circledR}\right)$. Der Partner der Patientin berichtet über Schnarchen und gelegentliche Atempausen. Nach der lleuseinleitung mit Propofol, Rocuronium und Sufentanil und Fortsetzung der Narkose mit Sevofluran wird die Beatmung aufgrund eines Bronchospasmus schwierig. Durch Vertiefung der Sevofluran-Narkose kann das Problem behoben werden. Beim Versuch, ein Kapnoperitoneum aufzubauen, treten jedoch nicht akzeptable Beatmungsprobleme auf und die Operation muss offen durchgeführt werden. Wegen des Verdachts auf ein Schlafapnoe-Syndrom und der intraoperativen Beatmungsprobleme wird beschlossen, die Patientin über Nacht im Aufwachraum zu überwachen. Die Patientin lehnt dies jedoch ab und wird zunehmend aggressiv und handgreiflich gegenüber dem Pflegepersonal. Ein Psychiater diagnostiziert schließlich ein Borderline-Syndrom vor dem Hintergrund wiederholten sexuellen Missbrauchs in der Kindheit mit nachfolgender Essstörung und Panikattacken. 
Bei OSAS mit Adipositas ist im Allgemeinen eine raumfordernde Zunahme des Fettgewebes bei normaler Anatomie der Atemwege für die Obstruktion verantwortlich. Bei ausgeprägtem OSAS werden die Atemwege bei Wachheit nur durch den Muskeltonus der Pharynxmuskulatur offen gehalten. Im Tiefschlaf und in Narkose erschlafft die Pharynxmuskulatur, und obstruktive Apnoen treten auf. Apnoen ziehen Hypoxämie und Hyperkapnie nach sich, bis der dadurch generierte Weckreiz zur Restabilisierung der Atemwege führt. Viele Patienten mit Adipositas entwickeln ein chronisches Hypoventilationssyndrom mit chronisch erhöhtem $\mathrm{pCO}_{2}$, Hypoxämie und schlafbezogener Atemstörung (in 90\% der Fälle ein OSAS) [3].

\section{Cave}

Die Inzidenz eines OSAS beträgt mit steigendem BMI bis zu $80 \%$ aller präoperativen Patienten mit Adipositas.

\section{INFO}

Kombination von OSAS und COPD

Die Kombination von chronisch obstruktiver Atemwegserkrankung (COPD) mit einem OSAS ist häufig verbunden mit Hypoxien bei Nacht, Hyperkapnie bei Tag und pulmonaler Hypertonie. Patienten mit diesem „Overlap Syndrome“ sind besonders durch perioperative respiratorische Komplikationen gefährdet [1].

\section{Lungenfunktionsstörungen}

Die Speicherung von Fett im Abdomen führt zu hohem intraabdominellem Druck, gegen den die Zwerchfellmuskulatur bei der Inspiration arbeiten muss. Fettgewebe über der Thoraxwand bedeutet darüber hinaus zusätzliche Atemarbeit für die thorakale Atemmuskulatur. Fetteinlagerung im Lungengewebe reduziert die Compliance der Lunge. Bei gleichbleibender Kraft der Atemmuskulatur wird dadurch das Atemzugvolumen reduziert und es tritt eine restriktive Atemstörung auf. Sinkt das Atemzugvolumen unter das Verschlussvolumen, entstehen Atelektasen, die zu intrapulmonalen Shunts und arterieller Hypoxie führen können. Außerdem erhöhen minderbelüftete Areale das Risiko für bronchopulmonale Infekte. Diese pathophysiologischen Effekte werden durch den bei Adipositas erhöhten Sauerstoffverbrauch mit entsprechend erhöhter Atemarbeit noch relativ verstärkt [4].

Adipositas führt zu inflammatorischen Veränderungen (s. Abschnitt „Inflammatorische Reaktion“), die auch das Lungengewebe betreffen - so auch zu einer erhöhten Inzidenz von Asthma bronchiale. Asthmaähnliche Auskultationsbefunde können allerdings auch vollständig durch adipositasbedingte Verengung der Atemwege hervor- gerufen werden. Entsprechend verschwinden die Symptome häufig nach Gewichtsverlust.

\section{DEFINITION}

\section{Obesitas-Hypoventilation}

Das Obesitas-Hypoventilationssyndrom (OHS, Pickwick-Syndrom) wird definiert durch die Kombination

- Hypoventilation, Hypoxyie und einer schlafassoziierten Atemstörung, meistens OSAS,

- Adipositas

- bei fehlender anderer Ursache für Hypoventilation

Die Symptome sind Atemnot, Tagesschläfrigkeit, morgendliche Kopfschmerzen und Depression.

\section{Herz-Kreislauf-Erkrankungen}

Viele Studien weisen darauf hin, dass der androide Typ der Adipositas mit einem höheren Risiko für kardiovaskuläre Erkrankungen assoziiert ist als der gynoide Typ [5].

Arterielle Hypertonie

Merke

Ansteigender BMI und die Prävalenz der Hypertonie korrelieren positiv.

Die hohe Prävalenz von arterieller Hypertonie bei Adipositas zieht außerdem ein erhöhtes Risiko für koronare Herzerkrankung, Herzinfarkt und generalisierte Arteriosklerose nach sich.

\section{Herzinsuffizienz}

Adipositas führt mit der Zeit auch zu Herzinsuffizienz: Etwa ein Drittel aller Patienten mit extremer Adipositas leidet unter einer adipositasinduzierten Kardiomyopathie. Kardiotoxische Effekte von Insulinresistenz, Steatosis, Sympathikusaktivierung, Hypoxie und Hyperkapnie können darüber hinaus zu pulmonaler Hypertonie [6] und zu einer Rechtsherzinsuffizienz führen.

\section{Herzrhythmusstörungen}

Mit steigendem Grad der Adipositas steigt die Inzidenz von Vorhofflimmern. Dies wird durch eine Dysfunktion des Sinusknotens und Infiltration des Reizleitungssystems durch Fettzellen erklärt. Auch die Inzidenz eines LongQT-Syndroms nimmt mit steigendem BMI zu und damit das Risiko einer Komplikation durch Serotonin-Antagonisten wie z. B. Ondansetron [1].

\section{Neurologische und psychische Erkrankungen}

Patienten mit Adipositas zeigen eine höhere Inzidenz für eine ganze Reihe von psychischen Erkrankungen. Darunter sind häufig Depressionen, Angsterkrankungen und Persönlichkeitsstörungen. Darüber hinaus können Diskri- 
minierung und daraus folgender gesellschaftlicher Ausschluss zu psychischen Problemen führen.

INFO

Psychische Erkrankungen

Gründe für die Gewichtszunahme bei psychischen

Erkrankungen sind [7]:

- psychiatrische Medikamente

- erhöhte Cortisolspiegel bei Depression

- Schlafstörungen mit Störung des Leptin-GhrelinVerhältnisses

- Dopaminmangel

- chronische Entzündung

Arterielle Hypertonie, Hyperlipidämie und Diabetes mellitus sind wahrscheinlich hauptsächlich dafür verantwortlich, dass Adipositas das Risiko für einen Schlaganfall deutlich erhöht.

\section{Muskuloskelettales System}

Adipositas führt häufig durch Belastung des muskuloskelettalen Systems zu Arthrose, Arthritis und Rückenschmerzen.

\section{Stoffwechsel}

Der androide Fettverteilungstyp ist oft mit dem metabolischen Syndrom (körperstammbetonte Adipositas, arterielle Hypertonie, Insulinresistenz und Hypercholesterinämie) verbunden. Dieser Verteilungstyp hat ein besonders hohes Risiko für Diabetes mellitus, koronare Herzkrankheit und perioperative Komplikationen. Adipositas führt aber auch unabhängig vom Fettverteilungstyp zu erhöhter Insulinresistenz. Diabetes mellitus Typ 2 tritt daher bei Adipositas überdurchschnittlich häufig auf.

Merke

Das Risiko für Diabetes mellitus steigt mit Schwere und Dauer der Adipositas.

Für das erhöhte Risiko wird u. a. die inflammatorische Reaktion durch Adipositas verantwortlich gemacht. Nach Magenbypass-Operation sinkt der Insulinbedarf sofort, sodass Antidiabetikadosierungen und Insulindosen angepasst werden müssen.

Das Körpergewicht korreliert positiv mit den Triglyzeridund Cholesterinspiegeln im Serum, weil Triglyzeride und Cholesterin insbesondere aus den abdominellen Fettspeichern freigesetzt werden.

\section{Blutgerinnung}

Patienten mit Adipositas haben ein erhöhtes Risiko für Thrombosen mit entsprechend erhöhtem Risiko für thromboembolische Erkrankungen wie Herzinfarkt, zere- brale Ischämie und Lungenembolien [8] (s. „Fallbeispiel Lungenembolie“).

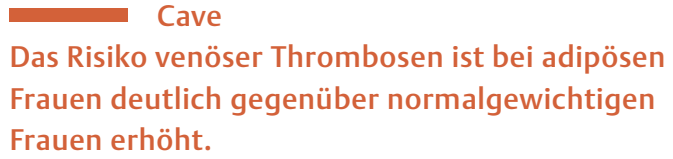

Deshalb ist es sinnvoll, die Thromboembolieprophylaxe bei Adipositas in Abhängigkeit von Operation und BMI länger aufrechtzuerhalten als sonst. Bei der manchmal auftretenden relativen Mangelernährung (s. Abschnitt „Gastrointestinales System“) mit Vitamin-K-Mangel kann die Gerinnung aber auch beeinträchtigt sein [1].

\section{FALLBEISPIEL \\ Lungenembolie}

Eine 52-jährige Patientin mit Adipositas (Gewicht 177 kg, Größe $161 \mathrm{~cm}$, Zustand nach Apoplex mit leichter Armparese links, Diabetes mellitus) wird wegen des Verdachts auf Appendizitis operiert. Postoperativ tritt im Aufwachraum eine niedrige Sauerstoffsättigung auf ( $\mathrm{SpO}_{2} 83 \%$ unter Raumluft). Anleitung zum tiefen Durchatmen, Aufrichten des Oberkörpers und Atemunterstützung mit CPAP (kontinuierlicher positiver Atemwegsdruck) bei Verdacht auf Atelektasen verändern die Situation nur geringfügig. Im Verlauf klagt die Patientin über Luftnot und dann über perakute Thoraxschmerzen. Ein EKG zeigt einen neu aufgetretenen Rechtsschenkelblock, die transthorakale Echokardiografie eine Rechtsherzbelastung. Nebenbefundlich wird eine Fettleber diagnostiziert. Im Spiral-CT des Thorax werden multiple Lungenembolien identifiziert, die Beinvenensonografie ergibt eine tiefe Beinvenenthrombose als Emboliequelle. Nachträglich berichtet die Patientin über schon länger bestehende Beschwerden in den Beinen. Sie habe diese jedoch auf ihre im Rahmen ihres Diabetes mellitus aufgetretenen Hautveränderungen an den Unterschenkeln und Füßen zurückgeführt.

\section{Inflammatorische Reaktion}

Die Hypertrophie des Fettgewebes kann dazu führen, dass die Blutversorgung der Fettzellen durch die vorhandenen Blutgefäße nicht mehr ausreicht [6]. In der Folge kommt es zu chronischer Gewebshypoxie und Zelltod [9]. Diese Veränderungen führen zur Abgabe von Adipokinen und Entzündungsmarkern wie TNF- $\alpha$ und IL- 6 durch Gewebsmakrophagen. Daraus folgt letztlich eine allgemeine inflammatorische Reaktion mit Aktivierung des sympathischen Nervensystems und des Renin-AngiotensinSystems.

\section{Gastrointestinales System}

Der erhöhte intraabdominelle Druck führt bei Adipositas auch zu erhöhtem intragastralem Volumen und Refluxerkrankung mit den entsprechenden Folgen. Daher muss nach diesen Problemen und ihrer Ausprägung bei der Anamnese gezielt gefragt und das Ergebnis bei der Nar- 
koseeinleitung z. B. durch Ileuseinleitung berücksichtigt werden.

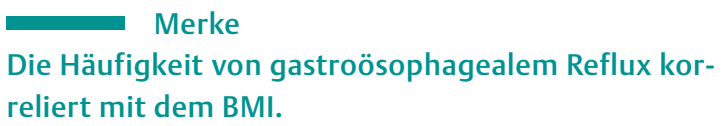

Die übermäßige Kalorienzufuhr bei Adipositas kann von einer selektiven Mangelernährung für Vitamin D, Magnesium, Phosphat, Eisen und Vitamin A begleitet werden. Niedrige Plasmaspiegel von Vitamin D sind mit einem erhöhten Risiko für Bluthochdruck, Diabetes und kardiovaskuläre Erkrankungen verbunden.

\section{Nieren und Harnwege}

Auch chronische Niereninsuffizienz tritt bei Adipositas häufiger auf als bei normalgewichtigen Patienten.

\section{PRAXISTIPP}

Bei extrem hohem Körpergewicht wird empfohlen, zur Bestimmung der glomerulären Filtrationsrate keine körpergewichtsabhängigen Formeln zu verwenden, sondern Clearance-Verfahren zur Bestimmung der Nierenfunktion mittels 24-h-Sammelurin anzuwenden.

\section{Leber und Gallenwege}

Die Fetteinlagerung in der Leber bei Adipositas (Fettleber) führt wie im sonstigen Körperfett zu inflammatorischen Reaktionen. In 15-20\% entwickelt sich daraus eine Leberzirrhose mit dem Risiko von Leberkarzinomen, portaler Hypertension, Aszites und Leberversagen. Außerdem verändert die Verfettung der Leber die Metabolisierung von Medikamenten in der Leber durch pathologische Expression und Aktivität von Leberenzymen. Trotzdem gibt es derzeit keine Empfehlungen, deswegen die Dosierung von Anästhetika zu verändern [6].

\section{Probleme nach Adipositas-Chirurgie}

Nach Magenverkleinerung kann eine postoperative Polyneuropathie aufgrund von Vitamin- und Ernährungsdefiziten auftreten. Die Symptome sind Erbrechen, Reflexabschwächung und Muskelschwäche. Ein verstellbares Magenband kann zu Störungen der Ösophagusmotilität und Dilatation proximal des Bandes führen, die auch nach dem Lösen des Bandes persistieren kann. Dadurch kann es trotz normaler präoperativer Nüchternheit zur Aspiration von Nahrungsbestandteilen kommen.

Cave

Ein akuter Notfall ist die Dislokation des Magenbandes mit Ischämie des Magens durch Gefäßkompression [1].
Es sollte mit dem Chirurgen besprochen werden, ob ein Magenband zur Narkoseeinleitung gelöst und eine Magensonde gelegt werden sollte. Dislokation des Bandes kann zu Magenischämie mit -perforation führen und macht sich durch Dysphagie und Oberbauchschmerzen bemerkbar [4].

\section{Pharmakokinetik}

\section{Allgemeine Aspekte}

Im Allgemeinen müssen auch bei übergewichtigen Patienten bei der Dosierung von Pharmaka das Verteilungsvolumen und die Clearance berücksichtigt werden. Das Verteilungsvolumen von Pharmaka hängt von der Fettlöslichkeit des Medikaments ab. Hydrophile Medikamente lagern sich nur wenig im Fettgewebe ein. Daher wird im Allgemeinen bei diesen Substanzen das ideale Körpergewicht des Patienten zur Dosisbestimmung für Einzeloder Aufsättigungsdosierungen vor Dauerinfusionen verwendet. Einzel- oder Aufsättigungsdosierungen lipophiler Medikamente wurden dagegen bisher nach dem tatsächlichen Gewicht berechnet [10]. Dies führt jedoch mit steigendem Körpergewicht zu kreislaufrelevanten Nebenwirkungen. Bei Adipositas nehmen außerdem auch die fettfreie Masse und die Verstoffwechselungsrate von Pharmaka zu. Diese Tatsache wird durch das Adjusted Body Weight (ABD, s. „Definition - Gewichtsmaße“) berücksichtigt.

\section{DEFINITION \\ Gewichtsmaße \\ - Body-Mass-Index (BMI): Body-Mass-Index $($ BMI $)=\frac{\text { Gewicht }[\mathrm{kg}]}{(\text { Größe }[\mathrm{m}])^{2}}$}

- Ideales Körpergewicht (Ideal Body Weight, IBW): IBW $=22 \times(\text { Größe }[\mathrm{m}])^{2}$

- Fettfreies Körpergewicht (Lean Body Weight, LBW, Männer): LBW $=\frac{9270 \times \text { Körpergewicht }}{6680+(216 \times \text { BMI })}$

- Fettfreies Körpergewicht (Lean Body Weight, LBW, Frauen): $\mathrm{LBW}=\frac{9270 \times \text { Körpergewicht }}{8780+(244 \times \mathrm{BMI})}$

- Angepasstes Körpergewicht (Adjusted Body Weight, $A B W)$ :

$\mathrm{ABW}=\mathrm{IBW}+0,4 \times($ Körpergewicht $-\mathrm{IBW})$

Von der Association of Anaesthetists of Great Britain and Ireland Society for Obesity and Bariatric Anaesthesia (AAGBI) wurden Empfehlungen herausgegeben, welche Maße bei der Dosierung von Anästhetika zugrunde gelegt werden sollten [4] (॰ Tab. 2). 


\section{Inhalationsanästhetika}

Moderne volatile Anästhetika sind prinzipiell aus pharmakokinetischer Sicht Injektionsnarkotika für die Aufrechterhaltung der Narkose bei Adipositas überlegen. Die am häufigsten verwendeten Gase Sevofluran und Desfluran werden wenig metabolisiert, haben eine geringe Löslichkeit im Blut und reichern sich wenig im Fettgewebe an. Sie bieten theoretisch gerade bei adipösen Patienten eine Reihe von Vorzügen.

Target Controlled Infusion (TCl) ist über $150 \mathrm{kgKG}$ (Marsh) und ab einem BMI von 35 für Frauen und 42 für Männer (Schneider) nicht mehr zugelassen [4].

\section{Bauliche und technische Voraussetzungen}

Viele Bau- und Einrichtungselemente von Krankenhäusern sind nur bis an bestimmte Grenzen für die Versorgung von extrem adipösen Patienten geeignet. Ab einem Körpergewicht von etwa $150 \mathrm{~kg}$ muss im Allgemeinen an die begrenzte Belastbarkeit der meisten Krankenhausbetten, sanitären Einrichtungen, Umlagerungshilfen und Operationstische gedacht werden. Gegebenenfalls müssen Schwerlasttische und Spezialbetten verwendet werden. Zusätzliches Material für die anästhesiologische Versorgung adipöser Patienten (z. B. Punktionsnadeln adäquater Länge) muss dabei verfügbar sein [4]. Gelkissen für die Lagerung der Extremitäten sind sehr hilfreich.

\section{Präoperative Evaluierung}

\section{Cave}

Patienten mit Adipositas vom androiden Fettverteilungstyp und Patienten mit metabolischem Syndrom haben ein besonders hohes Narkoserisiko.

Idealerweise erfolgt die präoperative Evaluierung schon Wochen vor dem Eingriff. 2-6 Wochen spezifische Diät können schon die Lungenfunktion verbessern. So lange vor der Operation bleibt auch noch genug Zeit, um ein behandlungsbedürftiges OSAS zu diagnostizieren [1]. Wahrscheinlich kann eine funktionelle Optimierung die Inzidenz von perioperativen Komplikationen verringern [11]. Daher wird für diese Patienten die Betreuung in spezialisierten Zentren empfohlen [6].

Prinzipiell sind auch bei Adipositas die Empfehlungen zur präoperativen Evaluation von Risikopatienten der DGAI und des BDA zu berücksichtigen. Die Erhebung klinischer Risikofaktoren in der Anamnese wird allerdings durch die Adipositas erschwert.
- Tab. 2 Dosierung von Anästhetika nach totalem, fettfreiem und angepasstem Körpergewicht (modifiziert nach [4]).

\begin{tabular}{|c|c|c|}
\hline $\begin{array}{l}\text { totales } \\
\text { Körpergewicht } \\
\text { (Total Body } \\
\text { Weight, TBW) }\end{array}$ & $\begin{array}{l}\text { fettfreies } \\
\text { Körpergewicht } \\
\text { (Lean Body Weight, } \\
\text { LBW) }\end{array}$ & $\begin{array}{l}\text { angepasstes } \\
\text { Körpergewicht } \\
\text { (Adjusted Body } \\
\text { Weight, ABW) }\end{array}$ \\
\hline - Succinylcholin & $\begin{array}{l}\text { - Propofol (Einleitung) } \\
\text { - Thiopental } \\
\text { - Fentanyl } \\
\text { - Rocuronium } \\
\text { - Atracurium } \\
\text { - Vecuronium } \\
\text { - Morphin } \\
\text { - Paracetamol } \\
\text { - Bupivacain } \\
\text { - Lidocain }\end{array}$ & $\begin{array}{l}\text { - Propofol } \\
\text { (Dauerinfusion) } \\
\text { - niedermoleku- } \\
\text { lares Heparin } \\
\text { - Alfentanil } \\
\text { - Neostigmin } \\
\text { (maximal } 5 \mathrm{mg} \text { ) } \\
\text { - Antibiotika }\end{array}$ \\
\hline
\end{tabular}

\section{Cave}

Da Patienten mit Adipositas sich körperlich wenig belasten, können trotz manifester koronarer Herzkrankheit typische Symptome in der Anamnese fehlen.

Symptome wie Belastungsdyspnoe und Thoraxschmerz können andererseits bei Adipositas auch ohne Herzerkrankung auftreten. Ödeme der Unterschenkel können z. B. bei Adipositas auch ohne Herzinsuffizienz vorliegen. Umso wichtiger ist die erweiterte apparative Diagnostik bei diesen Patienten in Ergänzung zum EKG [1]. Diese ist aber wegen Beschränkungen des zulässigen Körpergewichts bei der Anwendung vieler medizintechnischer Verfahren nur eingeschränkt möglich. Außerdem ist die Messung mit nichtinvasiven Verfahren wie der Echokardiografie aufgrund von Fettschichten schwierig.

\section{Obstruktives Schlafapnoe-Syndrom}

Ein OSAS sollte bei Adipositas nach einem Algorithmus ausgeschlossen werden [4]. Die präoperative Untersuchung im Schlaflabor aller Patienten mit Indikation zu bariatrischer Chirurgie wurde von verschiedenen Autoren empfohlen. Weniger aufwendig ist das Screening mittels eines für adipöse Patienten validierten Fragebogens (z. B. STOP-Bang Questionnaire). Bei positivem Test ist eine Untersuchung im Schlaflabor indiziert, die bei entsprechender Diagnose und Schwere des OSAS die Verordnung einer CPAP-Therapie oder anderer Hilfsmittel nach sich zieht [12]. Während Patienten mit nicht diagnostiziertem OSAS oder OSAS ohne CPAP-Therapie ein hohes Komplikationsrisiko haben, ist das Risiko bei Patienten mit gut therapiertem OSAS deutlich geringer [13]. Eine evtl. bereits etablierte CPAP-Therapie wird perioperativ fortgeführt, das gilt insbesondere ab dem Zeitpunkt der Verabreichung von Sedativa [1]. 


\section{Merke}

Auch bei negativem STOP-Bang Questionnaire sollte das Vorliegen von Belastungsdyspnoe, morgendlichen Kopfschmerzen und Zeichen der rechtsventrikulären Belastung im EKG zum Ausschluss eines OSAS führen [4].

Die Diagnose eines OSAS bei Adipositas sollte dazu veranlassen, nach kardiovaskulären Folgeerkrankungen zu suchen [1].

\section{Prämedikation}

Allgemeine prophylaktische Maßnahmen gegen Thromboembolien sind die Verwendung von Thrombosestrümpfen und die frühe Mobilisierung [14]. Aufgrund der hohen Inzidenz von Diabetes mellitus Typ 2 sollte der Blutzucker morgens präoperativ auch bei Patienten ohne bekannten Diabetes bestimmt werden. So kann eine Hyperglykämie erkannt und ggf. behandelt werden [1]. Die Gabe von Antazida ist sinnvoll, um die Azidität des Mageninhalts zu reduzieren.

PRAXISTIPP

Aufgrund des generell erhöhten Risikos für eine Schlafapnoe muss bei Patienten mit Adipositas auf Benzodiazepine am Vorabend der Operation verzichtet werden.

\section{Perioperatives Management}

\section{Regionalanästhesie}

Wegen der hohen Prävalenz respiratorischer Probleme bei Patienten mit Adipositas wird generell empfohlen, Operationen bei diesen Patienten wenn möglich in Regionalanästhesie durchzuführen. Es existiert jedoch keine klare Evidenz für die Überlegenheit der Regionalanästhesie gegenüber Allgemeinanästhesie [4].

Die Inzidenz schwieriger Punktionen ist aufgrund der Fettschichten höher als bei normalgewichtigen Patienten. Häufig werden längere Nadeln benötigt, um die Zielstrukturen zu erreichen $[4,15]$. Entsprechend höher ist auch der Bedarf an erfahrenen Anästhesisten [4]. Aufgrund des hohen Fettgehalts im Epiduralraum herrscht dort ein relativ hoher Druck. Daher wird von einigen Autoren empfohlen, bei Epiduralanästhesie die Dosierung der Lokalanästhetika zu reduzieren, um eine zu starke kraniale Ausbreitung zu verhindern.

\section{Narkoseeinleitung}

Die Narkoseeinleitung sollte bei besonders komplexen Fällen im Operationssaal erfolgen, weil so Transport- und Umlagerungsprobleme vermieden werden. Bei Allge- meinanästhesie ist der Einsatz von kurzwirksamen Substanzen wie Desfluran und Remifentanil sinnvoll. Succinylcholin sollte vermieden werden, weil die dadurch verursachten Muskelfaszikulationen den Sauerstoffverbrauch erhöhen.

\section{Gefäßpunktionen}

Bei schwierigen Punktionsbedingungen für periphere Venenkatheter ist ein Sonografiegerät beim Aufsuchen der Venen nützlich. Auch ungewöhnliche Punktionsorte sollten berücksichtigt werden und mindestens 2 Venenwege gelegt werden [4]. Bei sehr schlechten Venenverhältnissen kann es notwendig werden, einen zentralen Venenkatheter zu legen; auch hierfür sollte ein Sonografiegerät zur Verfügung stehen.

\section{Atemwegsmanagement}

\section{Merke}

Aufgrund der vergleichsweise geringen funktionellen Residualkapazität adipöser Patienten ist bei ihnen die Sauerstoffreserve in der Lunge gegenüber normalgewichtigen Patienten reduziert.

Adäquate Präoxygenierung ist besonders wichtig, weil bei Adipösen die Sauerstoffsättigung des Hämoglobins besonders schnell abfällt. Die umgekehrte Trendelenburg-Lagerung mit einer Kippung des OP-Tisches um ca. $30^{\circ}$ reduziert den Druck des Abdomens auf die Lunge [4]. Dadurch wird die inspiratorische Atemarbeit reduziert und das Risiko der Bildung von Atelektasen verringert.

\section{Cave \\ Wenn nur der Oberkörper hoch gelagert wird, kann sich der Druck des Abdomens auf die Lunge erhöhen, weil der Bauch beim Anwinkeln der Beine zusam- mengedrückt werden kann.}

Eine noch bessere Atelektasenvorbeugung, bessere Oxygenierung und längere Apnoetoleranz kann durch CPAP oder nichtinvasive Beatmung für etwa 5 Minuten vor Narkoseeinleitung erreicht werden [16].

Während früher grundsätzlich eine Ileuseinleitung bei Adipositas empfohlen wurde, wird dies inzwischen kontrovers diskutiert [17]. Dies wird analog zum Vorgehen in der Kinderanästhesie damit begründet, dass durch die geringe Apnoetoleranz auch ein hohes Hypoxierisiko bei der Ileuseinleitung besteht. Bei Patienten ohne zusätzliche Risikofaktoren für Aspiration ist es deshalb praktikabel,

- nach Präoxygenierung die Narkose einzuleiten,

- nach Erreichen ausreichender Narkosetiefe einen Guedel-Tubus einzulegen und

- den Patienten vorsichtig (ggf. druckkontrolliert maschinell) über eine Gesichtsmaske zu beatmen, bis ausreichende Muskelrelaxierung erreicht ist. 
Cave

Maskenbeatmung ist bei Adipositas oft nicht einfach.

Relativ häufig werden bei Adipositas Zeichen für einen schwierigen Atemweg festgestellt. Mundöffnung und Beweglichkeit der Halswirbelsäule werden durch perimandibuläre und nuchale Fettpolster reduziert, das Volumen von Mundhöhle und Rachen wird durch Fetteinlagerung in die Weichteile verkleinert (s. „Info - Schwierige Intubation“). Geringe Unterkiefermobilität, Nackenfettpolster, OSAS, Schnarchen und Adipositas gelten als unabhängige Prädiktoren des schwierigen Atemwegs. Folglich ist es sinnvoll, schon vor der Einleitung die notwendigen Hilfsmittel einsatzbereit greifbar und einen individuellen Plan zum Management des schwierigen Atemwegs besprochen zu haben. Besonders effektiv ist die prophylaktische Bereitstellung eines Videolaryngoskops.

\section{INFO}

\section{Schwierige Intubation}

Adipositas ist mit gegenüber normalgewichtigen Patienten erhöhtem Risiko für schwierige Intubation assoziiert. Zusätzlich zu den üblichen Kriterien kann ein Halsumfang von mehr als $60 \mathrm{~cm}$ auf eine schwierige Intubation hinweisen. Der Querschnitt der Trachea korreliert negativ mit dem BMI.

Der Einsatz von Larynxmasken bei Adipositas ist umstritten. Wenn man sich für die Verwendung einer Larynxmaske entscheidet, erscheint es aufgrund theoretischer Überlegungen sinnvoll, eine Larynxmaske mit der Möglichkeit zur Einlage einer Magensonde zu verwenden.

\section{Monitoring}

Die Überwachung von Patienten mit Adipositas ist technisch schwieriger als die Überwachung normalgewichtiger Patienten. Die nichtinvasive Blutdruckmessung kann bei voluminösen Extremitäten sehr unzuverlässig werden, und meistens sind besonders breite Blutdruckmanschetten notwendig.

\section{PRAXISTIPP}

Insbesondere bei angelagerten Armen ist die Indikation zur invasiven Blutdruckmessung großzügig zu stellen, weil Korrekturen der Blutdruckmanschettenanlage intraoperativ sehr umständlich sind.

Thorakale Fettschichten können die im EKG gemessene Spannung durch Isolation deutlich reduzieren und dadurch vor allem die ST-Strecken-Analyse erschweren. Der zentrale Venendruck (ZVD) wird bei Adipositas erheblich durch den auf die $V$. cava inferior und den gesamten Thorax übertragenen hohen intraabdominellen Druck beeinflusst. Deshalb muss bei der Interpretation des ZVD noch mehr als sonst beachtet werden, dass nur Veränderungen im Verlauf interpretiert werden können. Bei Verwendung von auf der Pulskonturanalyse basierenden, nichtinvasiven oder wenig invasiven Verfahren zur Messung von Herzzeitvolumen und Vorlastparametern muss beachtet werden: Diese Verfahren wurden nicht für Patienten mit Adipositas validiert.

Adipositas ist mit einem erhöhten Risiko für Awareness insbesondere während der Narkoseeinleitung unter Verwendung von Muskelrelaxanzien verbunden [18]. Dies wird auf die schnelle Umverteilung eines Injektionsbolus ins Fettgewebe zurückgeführt [19]. Besonders bei einer TIVA in Kombination mit Muskelrelaxanzien wird deshalb empfohlen, die Narkosetiefe mit einem EEG-basierten Verfahren zu überwachen [4].

\section{Beatmung}

Wie bei den meisten Patienten ist bei Adipösen im Allgemeinen eine druckkontrollierte Beatmung mit moderaten Tidalvolumina $(6-8 \mathrm{ml} / \mathrm{kg})$ zur Vermeidung von Komplikationen sinnvoll - auch wenn die Datenlage hinsichtlich des Vergleichs von druckkontrollierter und volumenkontrollierter Beatmung inhomogen ist.

\footnotetext{
Merke

Die Berechnung des angestrebten Tidalvolumens erfolgt auf der Grundlage des idealen Körpergewichts (s. „Definition - Gewichtsmaße“). Denn die Zunahme der Körpermasse durch Fetteinlagerung geht nicht mit einer Vergrößerung der Lunge einher [1].
}

Die adäquate Einstellung des PEEP (positiver endexspiratorischer Druck) ist wegen der hohen Atelektaseneigung besonders wichtig. Optimal wäre es, auch in Narkose den individuell besten PEEP basierend auf $\mathrm{SaO}_{2}, \mathrm{paO}_{2}$, Compliance, arteriellem Blutdruck und Herzzeitvolumen zu ermitteln. Im Allgemeinen ist es ausreichend, einen PEEP zwischen 10 und $15 \mathrm{cmH}_{2} \mathrm{O}$ so einzustellen, dass eine adäquate Oxygenierung sichergestellt ist. Vor allem bei Eingriffen mit operationsbedingt zusätzlich erhöhtem intraabdominellem Druck (z. B. Laparoskopien) sind manchmal wiederholte Rekrutierungsmanöver nötig, um die Oxygenierung und das verfügbare Lungenvolumen zu verbessern [16].

Die Atemfrequenz muss bei Adipositas etwas höher als sonst eingestellt werden, weil aufgrund des hohen $\mathrm{O}_{2}$ Verbrauchs auch die $\mathrm{CO}_{2}$-Produktion erhöht ist. Bei der Auswertung von Blutgasanalysen muss berücksichtigt werden, dass adipöse Patienten eine höhere Differenz zwischen dem endtidal und arteriell gemessenen $\mathrm{CO}_{2}-$ Gehalt aufweisen [1]. 


\section{Narkoseausleitung}

Es gibt Hinweise darauf, dass der Verzicht auf hohe $\mathrm{O}_{2}$ Konzentrationen vor Narkoseausleitung zu geringerer Mikroatelektasenbildung und besserer postoperativer Lungenfunktion führt. Auch während der Ausleitung ist die umgekehrte Trendelenburg-Lage aufgrund des hohen intraabdominellen Drucks hilfreich [4].

Cave

Wurden Muskelrelaxanzien eingesetzt, muss eine evtl. Restrelaxierung durch quantitative Relaxometrie ausgeschlossen oder ggf. reversiert werden.

Vor der Extubation muss eine ausreichende Wachheit mit Rückkehr der Schutzreflexe sichergestellt sein. Bei Eingriffen im Bereich von Hals und Pharynx können Ödeme der pharyngealen Weichteile über die üblicherweise nach diesen Operationen erwarteten Veränderungen hinaus auftreten. Auch postoperativ kann vorübergehend eine prophylaktische nichtinvasive Atemunterstützung mit CPAP sinnvoll sein, und eine aktive Atemtherapie sollte routinemäßig eingesetzt werden [1].

\section{Postoperative Betreuung}

Die häufigsten postoperativen Komplikationen treten bei Adipositas noch öfter auf. Besonders wichtig ist die Verbesserung der Lungenfunktion durch Bevorzugung einer sitzenden Position, Atemtraining und Frühmobilisation. Sekundäre Verschlechterung der Lungenfunktion noch am 3. oder 4. postoperativen Tag mit Notwendigkeit von PEEP oder nichtinvasiver Beatmung wurde beschrieben [1].

Postoperativ müssen bestimmte Patienten bis zu ihrer Mobilisierung über Nacht kontinuierlich pulsoxymetrisch überwacht werden:

- Patienten mit OSAS ohne Atemhilfe (z. B. zu Hause Anwendung von (PAP) und

- Patienten mit Atemhilfe und zusätzlichen Risikofaktoren (z. B. schwieriger Atemweg) [4].

Die patienteneigene Atemhilfe sollte so bald wie möglich nach der OP wieder eingesetzt werden. Das OSAS wird perioperativ u. a. durch Opioide und Schlafstörungen verstärkt [1]. Nichtinvasive Beatmung direkt nach der Extubation kann die Letalität senken und ist eine sinnvolle Erstmaßnahme bei postoperativer Ateminsuffizienz, um eine Reintubation zu verhindern [20].

\section{Merke}

Die Verlegung aus dem Aufwachraum ist erst zu erwägen, wenn die normalen Verlegungskriterien erfüllt sind und über eine Stunde bei normaler Atemfrequenz keine Hypopnoen/Apnoen aufgetreten sind [4].
INFO

Thromboseprophylaxe

Zur Thromboseprophylaxe ist eine frühe Mobilisierung noch am OP-Tag sinnvoll [14]. Dazu gehört auch die frühe Entfernung von Blasenkatheter und Infusionsleitungen [1]. Die Effektivität konventioneller Kompressionsstrümpfe ohne intermittierende Kompression ist nur wenig belegt. Bei adipösen Patienten muss besonders darauf geachtet werden, dass keine Blutgefäße durch zu enge Strümpfe okkludiert werden. Empfehlungen zur Dosisanpassung der medikamentösen Prophylaxe wurden vom britischen Haemostasis, Anticoagulation and Thrombosis (HAT) Committee publiziert.

\section{Schmerztherapie}

Auch die postoperative Schmerztherapie bereitet bei Adipositas besondere Probleme. Wenn möglich, sollte eine Regionalanalgesie mit Katheterverfahren erfolgen. Opioide haben bei Patienten mit Adipositas eine besonders stark sedierende Wirkung und verstärken die Neigung zu einer Schlafapnoe. Umgekehrt haben Patienten mit diagnostiziertem oder vermutetem OSAS oder OHS ein erhöhtes Risiko für eine opioidinduzierte Atemdepression. Diese Patienten sollten deshalb nach Verabreichung von Opioiden - auch als patientenkontrollierte Analgesie für mindestens 24 Stunden kontinuierlich einschließlich Pulsoxymetrie überwacht werden. Mit adäquater Behandlung des OSAS durch das eigene CPAP-Gerät reduziert sich das Risiko. Die Anwendung des CPAP-Geräts kann aber postoperativ unmöglich sein, wenn im Bereich der Atemwege operiert wurde. Eine Überwachung auf der Intensivstation ist selten notwendig und hängt mehr von den Begleiterkrankungen als von der Adipositas ab [4].

\section{INFO}

\section{Ambulante Anästhesie}

Nach britischen Leitlinien ist bei einem $\mathrm{BMI}<40$ eine ambulante Anästhesie im Allgemeinen möglich [4]. Zum Vorgehen bei OSAS wird auf die im entsprechenden Abschnitt erwähnten Empfehlungen verwiesen. Ambulante Anästhesie ist bei OSAS nach Ansicht mancher Autoren möglich, wenn ein CPAP-Gerät einsetzbar ist, Begleiterkrankungen optimal therapiert sind und Nichtopioide für die Schmerztherapie ausreichen. Über ambulante laparoskopische Cholezystektomien und ambulante laparoskopische Magenbanding-Operationen wurde in der Literatur berichtet. Die ambulante Durchführung einer Anästhesie bei Adipositas sollte aber eine Einzelfallentscheidung mit sorgfältiger Risikoabwägung bleiben. 


\section{Fazit}

Die steigende Prävalenz der Adipositas stellt besondere Anforderungen an die perioperative anästhesiologische Betreuung. Dies betrifft sowohl die Kenntnis der Pathophysiologie und Pharmakokinetik als auch das Management der Folge- und Begleiterkrankungen der Adipositas. Dazu kommen die praktischen Probleme des Umgangs mit adipösen Patienten bei Transport und Lagerung. Am besten ist es, alle Maßnahmen zum perioperativen Vorgehen bei adipösen Patienten in einem standardisierten Protokoll zusammenzufassen. Ein in der Versorgung adipöser Patienten besonders erfahrenes Team mit entsprechender Leitung sollte verfügbar sein. Der BMI aller Patienten sollte im OP-Plan angegeben werden.

\section{KERNAUSSAGEN}

- Die Prävalenz der Adipositas hat in den letzten 10 Jahren auch in Deutschland weiter zugenommen.

- Hypertonie, Fettstoffwechselstörungen, Diabetes mellitus, kardiovaskuläre Erkrankungen und Schlafapnoe-Syndrom sind Folge- und Begleiterkrankungen der Adipositas.

- Die perioperative Betreuung adipöser Patienten erfordert spezielle räumliche, apparative und personelle Voraussetzungen.

- Probleme mit Atemwegsmanagement, Atmung und Monitoring sind bei Adipositas relativ häufig.

- Regionalanästhesiologische Verfahren sollten bevorzugt eingesetzt werden.

- Kurzwirksame Substanzen wie Desfluran und Remifentanil sind zu bevorzugen.

- Die Präoxygenierung wird durch umgekehrte Trendelenburg-Lagerung und Unterstützung der Atmung mit kontinuierlichem positivem Atemwegsdruck effektiver.

- Auch in der postoperativen Schmerztherapie sind Regionalanästhesieverfahren zu bevorzugen.

\section{Interessenkonflikt}

Priv.-Doz. Dr. Axel Fudickar hat Honorare für Vorträge von der Firma Medtronic erhalten. Prof. Dr. Berthold Bein hat Honorare für Vorträge und Beratungstätigkeit von Abbvie, Baxter und Air Liquide erhalten.

\section{Autorinnen/Autoren}

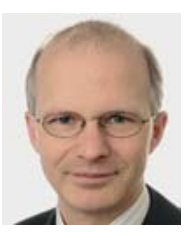

\section{Axel Fudickar}

Priv.-Doz. Dr. med., 1989-1995 Medizinstudium an der Georg-August-Universität Göttingen und der Christian-Albrechts-Universität zu Kiel. 1995-1997 AiP in der Universitäts-Kinderklinik Freiburg. Seit 1997 Klinik für Anästhesiologie und Operative Intensivmedizin, Universitätskli- nikum Schleswig-Holstein, Campus Kiel. 2004 Facharzt für Anästhesiologie. Seit 2010 Oberarzt. Schwerpunkte: Neuroanästhesie, klinische Neurophysiologie, Risikomanagement.

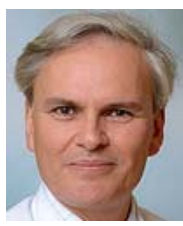

\section{Berthold Bein}

Prof. Dr. med., M. A., DEAA. Chefarzt der Abteilung für Anästhesiologie und Intensivmedizin, Asklepios Klinik St. Georg, Hamburg. Von 2009-2015 DGAI-Landesvorsitzender in Schleswig-Holstein. Schwerpunkte: hämodynamisches Monitoring, Antibiotic Stewardship und Patient Blood Management.

\section{Korrespondenzadresse}

Priv.-Doz. Dr. med. Axel Fudickar

Universitätsklinikum Schleswig-Holstein, Campus Kiel Klinik für Anästhesiologie und Operative Intensivmedizin Arnold-Heller-Straße 3, Haus 12

24105 Kiel

axel.fudickar@uksh.de

Wissenschaftlich verantwortlich gemäß Zertifizierungsbestimmungen

Wissenschaftlich verantwortlich gemäß Zertifizierungsbestimmungen für diesen Beitrag ist PD Dr. med. Axel Fudickar, Kiel.

\section{Literatur}

[1] De Jong A, Verzilli D, Geniez M et al. [Why is the morbidly obese patient at high risk of anesthetic complications?]. Presse Med 2018; 47: 453-463

[2] Robert Koch-Institut. Übergewicht und Adipositas, 2018. Im Internet: http://www.rki.de/DE/Content/Gesundheitsmonitoring/Themen/Uebergewicht_Adipositas/Uebergewicht_ Adipositas_node.html; Stand: 08.01.2019

[3] Chau EH, Lam D, Wong J et al. Obesity hypoventilation syndrome: a review of epidemiology, pathophysiology, and perioperative considerations. Anesthesiology 2012; 117: 188-205

[4] Nightingale CE, Margarson MP, Shearer E et al. Peri-operative management of the obese surgical patient 2015: Association of Anaesthetists of Great Britain and Ireland Society for Obesity and Bariatric Anaesthesia. Anaesthesia 2015; 70: 859-876

[5] Tchernof A, Despres JP. Pathophysiology of human visceral obesity: an update. Physiol Rev 2013; 93: 359-404

[6] Ortiz VE, Kwo J. Obesity: physiologic changes and implications for preoperative management. BMC Anesthesiol 2015; 15: 97

[7] Taylor VH, McIntyre RS, Remington G et al. Beyond pharmacotherapy: understanding the links between obesity and chronic mental illness. Can J Psychiatry 2012; 57: 5-12

[8] Gould MK, Garcia DA, Wren SM et al. Prevention of VTE in nonorthopedic surgical patients: Antithrombotic Therapy and Prevention of Thrombosis, 9th ed: American College of Chest Physicians Evidence-Based Clinical Practice Guidelines. Chest 2012; 141 (Suppl. 2): e227S-e277S

[9] Revelo XS, Luck H, Winer S et al. Morphological and inflammatory changes in visceral adipose tissue during obesity. Endocr Pathol 2014; 25: 93-101 
[10] Bein B, Hocker ], Fudickar A et al. [Anaesthesia management of the obese patient]. Anasthesiol Intensivmed Notfallmed Schmerzther 2009; 44: 600-608; quiz 610

[11] Lemanu DP, Srinivasa S, Singh PP et al. Optimizing perioperative care in bariatric surgery patients. Obes Surg 2012; 22: 979-990

[12] Chung F, Yang Y, Liao P. Predictive performance of the STOPBang score for identifying obstructive sleep apnea in obese patients. Obes Surg 2013; 23: 2050-2057

[13] Joshi GP, Ankichetty SP, Gan T] et al. Society for Ambulatory Anesthesia consensus statement on preoperative selection of adult patients with obstructive sleep apnea scheduled for ambulatory surgery. Anesth Analg 2012; 115: 1060-1068

[14] Mechanick JI, Youdim A, Jones DB et al. Clinical practice guidelines for the perioperative nutritional, metabolic, and nonsurgical support of the bariatric surgery patient - 2013 update: cosponsored by American Association of Clinical Endocrinologists, the Obesity Society, and American Society for Metabolic \& Bariatric Surgery. Surg Obes Relat Dis 2013; 9: 159-191

[15] Ingrande J, Brodsky JB, Lemmens HJ. Regional anesthesia and obesity. Curr Opin Anaesthesiol 2009; 22: 683-686

[16] Futier E, Constantin JM, Pelosi P et al. Noninvasive ventilation and alveolar recruitment maneuver improve respiratory func- tion during and after intubation of morbidly obese patients: a randomized controlled study. Anesthesiology 2011; 114: 1354-1363

[17] Freid EB. The rapid sequence induction revisited: obesity and sleep apnea syndrome. Anesthesiol Clin North America 2005; 23: 551-564, viii

[18] Pandit J], Andrade J, Bogod DG et al. The 5th National Audit Project (NAP5) on accidental awareness during general anaesthesia: protocol, methods and analysis of data. Anaesthesia 2014; 69: 1078-1088

[19] Ingrande J, Lemmens HJ. Dose adjustment of anaesthetics in the morbidly obese. Br J Anaesth 2010; 105 (Suppl 1): i16-i23

[20] Jaber S, De Jong A, Castagnoli A et al. Non-invasive ventilation after surgery. Ann Fr Anesth Reanim 2014; 33: 487-491

Bibliografie

DOI https://doi.org/10.1055/a-0636-2782

Anästhesiol Intensivmed Notfallmed Schmerzther 2019; 54: 242-254 ( Georg Thieme Verlag KG Stuttgart · New York ISSN 0939-2661 


\section{Punkte sammeln auf CME.thieme.de}

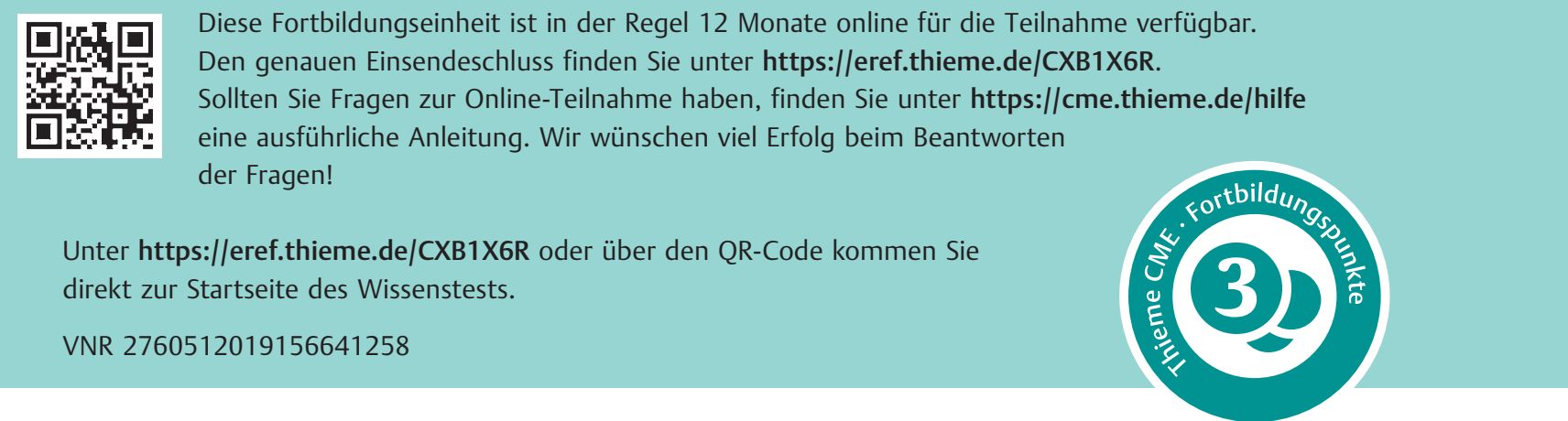

\section{Frage 1}

Wie ist die Adipositas nach der Weltgesundheitsorganisation (WHO) definiert?

A Body-Mass-Index $>35 \mathrm{~kg} / \mathrm{m}^{2}$

B Übermaß an Körperfett, welches zu unerwünschten Folgen für Gesundheit und Wohlbefinden führt

C Taillenumfang von $>115 \mathrm{~cm}$

D Körperfettanteil von mehr als 30\%

E Gewicht > Körpergröße - 100 (cm)

\section{Frage 2}

Wie hoch ist ungefähr die Prävalenz der Adipositas bei Männern in Deutschland?
A $15 \%$
B $20 \%$
C $25 \%$
D $30 \%$
E $35 \%$

\section{Frage 3}

Welche Aussage ist richtig? Das Schlafapnoe-Syndrom (obstruktives Schlafapnoe-Syndrom, OSAS) wird diagnostiziert bei ...

A mehr als 3 Schnarchepisoden von mehr als 5 Minuten Länge und Einschlafen am Tag.

B mehr als 2 Apnoe- oder Hypopnoe-Phasen pro Stunde Schlaf in Kombination mit Schnarchen.

C mehr als 5 Wachliegephasen pro Nacht und Einschlafen vor dem Fernseher abends.

D mehr als 5 Apnoe- oder Hypopnoe-Phasen pro Stunde Schlaf in Kombination mit Tagesmüdigkeit.

E mehr als 5 Konzentrationsstörungen pro Stunde Wachheit in Kombination mit Durchschlafstörung.

\section{Frage 4}

Welche Aussage zum Obesitas-Hypoventilationssyndrom ist falsch?

A Man nennt das Syndrom auch Pickwick-Syndrom.

B Ein Symptom sind morgendliche Kopfschmerzen.

C Zur Definition gehören chronisch über $6 \mathrm{kPa}$ erhöhter $\mathrm{PaCO}_{2}$ und eine schlafassoziierte Atemstörung.

D Die Erkrankung tritt häufig zusammen mit einem obstruktiven Schlafapnoe-Syndrom auf.

E Das Syndrom tritt typischerweise bei Übergewicht (BMI 25$30 \mathrm{~kg} / \mathrm{m}^{2}$ ) auf.

\section{Frage 5}

Was ist kein Grund für die Gewichtszunahme bei psychischen Erkrankungen?

A erniedrigte Cortisolspiegel bei Depression

B Schlafstörungen mit Störung des Leptin-Ghrelin-Verhältnisses

C chronische Entzündung

D psychiatrische Medikamente

E Dopaminmangel

\section{Frage 6}

Welche Aussage zu den Fettverteilungstypen ist richtig?

A Der nach Peter Paul Rubens benannte Rubens-Typ hat ein besonders erhöhtes kardiopulmonales Risiko.

B Beim androiden Typ ist das Fett besonders an Hüften und Oberschenkeln lokalisiert.

C Den Rubens-Typ nennt man nach der runden, bauchbetonten Fettverteilung an Frauenkörpern in seinen Gemälden auch Apfel-Typ.

D Der androide Typ heißt auch Falstaff-Typ nach John Falstaff aus Shakespeares Dramen.

E Der Birnen-Typ zeigt eine typische birnenförmige Fettverteilung im Bereich von Kopf und Hals.

\section{- Weitere Fragen auf der folgenden Seite...}




\section{Punkte sammeln auf CME.thieme.de}

Fortsetzung $\ldots$

\section{Frage 7}

Welche Aussage zur Narkoseeinleitung bei Adipositas ist richtig?

A Eine lleuseinleitung ist obligat.

B Der Oberkörper sollte flach gelagert werden.

C Durch Umverteilung von Injektionsnarkotika ins Fettgewebe kann Awareness auftreten.

D Der Halsumfang hat keine Bedeutung für die Intubationsbedingungen.

E Schwierige Venenpunktion ist bei Adipositas selten.

\section{Frage 8}

Welche Medikamentendosierung während Allgemeinanästhesie bei Adipositas ist nicht sinnvoll?

A Dosierung der Propofol-Dauerinfusion orientierend am angepassten Körpergewicht (Adjusted Body Weight, ABW)

B Dosierung der Succinylcholin-Gabe orientierend am aktuellen Körpergewicht

C Dosierung der Rocuronium-Gabe orientierend am fettfreien Körpergewicht (Lean Body Weight, LBW)

D Dosierung der Alfentanil-Gabe orientierend am angepassten Körpergewicht (Adjusted Body Weight, ABW)

E Dosierung einer Antibiotika-Gabe orientierend am aktuellen Körpergewicht

\section{Frage 9}

Welche Maßnahme während Allgemeinanästhesie bei Adipositas ist sinnvoll?

A Einstellen eines möglichst niedrigen PEEP, um ein ausreichendes Tidalvolumen zu erreichen

B Rekrutierungsmanöver bei Abfall der Sauerstoffsättigung und Verdacht auf Atelektasenbildung

C Kopftieflage zur Aspirationsprophylaxe während der Operation

D möglichst hohe inspiratorische Sauerstoffkonzentrationen, um den erhöhten Sauerstoffbedarf zu kompensieren

E angestrebtes Tidalvolumen nach dem aktuellen Körpergewicht berechnen $(6-8 \mathrm{ml} / \mathrm{kg})$

\section{Frage 10}

Durch welche Intervention kann die Atmung des adipösen Patienten im Aufwachraum regelmäßig verbessert werden?
A stabile Seitenlage
B Oberkörperhochlage und Atemtraining
C Aufforderung zu schonender flacher Atmung
D prophylaktische hoch dosierte Sauerstoff-Gabe
E Bevorzugung von intravenöser Analgesie gegenüber Regio- nalanästhesieverfahren

